\title{
EOSINOPHILIC GASTROENTERITIS PRESENTING WITH ASCITES; CASE REPORT AND REVIEW OF LITERATURE \\ Hany M. Elsadek
}

Lecturer of internal medicine, gastroenterology unit, internal medicine department, faculty of medicine, Zagazig

University, Egypt

Eosinophilic gastroenteritis (EGE) is an uncommon disorder affecting both children and adults, that is characterized by eosinophilic infiltration of the stomach and intestine with or without peripheral blood eosinophilia, in the absence of other known causes of eosinophilia. Presentation may vary depending on the location as well as on the depth and extent of involvement and usually runs a chronic relapsing course. Eosinophilic ascites (EA) is probably the most unusual and rare presentation of EGE. Almost all reported cases of EA are idiopathic. One case of EGE presenting with EA, that was successfully diagnosed and treated, is reported here. The subsequent review describes the main features and management of EGE and EA.

Keywords: eosinophilia, gastroenteritis, ascites.

\section{INTRODUCTION}

$\mathbf{E}$ osinophilic gastrointestinal disorders (EGID) is the set of diseases affecting primarily gastrointestinal tract, from the esophagus to the rectum, characterized by an abnormal infiltrate of eosinophils, in the absence of other known causes of eosinophilia. The parts most frequently affected are the stomach and small intestine, that is called eosinophilic gastroenteritis (EGE). Eosinophilic ascites (EA) is a rare presentation of EGE and its diagnosis is often difficult due to lack of specific characteristics and histological confirmation is always necessary. Most reported cases of EA are idiopathic, often accompanied by an atopic condition, such as asthma, or food allergy. ${ }^{1,2}$

\section{CASE REPORT}

A 41 years old Saudi male patient was admitted in the department of internal medicine in Royal Commission Medical Center (RCMC), Yanbu, KSA, for investigation of a recurrent severe crampy upper abdominal pain for three months. The pain was not related to meals, not accompanied with vomiting, diarrhea or fever. The patient was completely free of pain in between the painful episodes. However, over the last few weeks before admission, he suffered from poor appetite, lost five $\mathrm{kg}$ of body weight and also, noticed progressive abdominal distension. The patient mentioned that he had infrequent mild episodes of similar abdominal pain over the past several years. The patient gave history of childhood asthma. The patient had no history of allergic rhinitis, food allergy, atopic dermatitis, or any other systemic disease. On admission, his body temperature was $36.2^{\circ} \mathrm{C}$, blood pressure was $124 / 82$ $\mathrm{mmHg}$, respiratory rate was $18 / \mathrm{min}$, heart rate was $68 \mathrm{bpm}$ and body weight was $64 \mathrm{~kg}$. His physical examination showed moderate abdominal distention with shifting dullness. There were no abdominal tenderness or organomegally. Examination of the chest, heart and nervous system were unremarkable. There were no lower limb edema or peripheral lymphadenopathy.

Laboratory investigations were as follows: Complete blood count (CBC): white blood cells (WBCs) $11.7 \times 10^{9} / \mathrm{L} ; \quad$ neutrophils, $2.19 \times 10^{9} / \mathrm{L}$ (18.7\%); lymphocytes, $2.76 \times 10^{9} / \mathrm{L} \quad(23.55 \%)$; monocytes, $\quad 0.48 \times 10^{9} / \mathrm{L} \quad(4.13 \%)$; eosinophils, $6.19 \times 10^{9} / \mathrm{L} \quad(52.95 \%) ; \quad$ basophils, $0.08 \times 10^{9} / \mathrm{L}$ $(0.66 \%)$; red blood cells (RBCs) $4.94 \times 10^{12} / \mathrm{L}$; hemoglobin, $13.3 \mathrm{~g} / \mathrm{dl}$; and platelets $440 \times 10^{9} / \mathrm{L}$. Peripheral hypereosinophilia was documented on repeated CBC. Blood urea, serum creatinine, serum sodium, serum potassium, alanine aminotransferase, aspartate aminotransferase, serum albumin, total protein, total bilirubin, TSH, total cholesterol, triglycerides, fasting blood glucose, ESR, CRP, serum CEA, CA 19-9, CA 125, C3, C4 and antinuclear antibody (ANA) were all normal. The serum IgE was elevated $(2319 \mathrm{IU} / \mathrm{mL})$, while $\mathrm{IgG}$, IgA, and IgM levels were within normal ranges. $\mathrm{HCV}-\mathrm{Ab}$ and HBsAg were negative. Stools analysis was unremarkable with no detectable parasites or ova, and urinalysis was normal. A skin-prick test (SPT) was negative. Ultrasound guided Ascitic tapping revealed: turbid, orange fluid. Analysis of ascitic aspirate showed protein level $45 \mathrm{~g} / \mathrm{L}$ and albumin $24 \mathrm{~g} / \mathrm{dL}$. Cell counts were: WBCs, $7.4 \times 10^{9} / \mathrm{L}$; neutrophils, 8\%; lymphocytes, $2 \%$; eosinophils, $90 \%$ (the absolute eosinophil count was $\left.6.67 \times 10^{9} / \mathrm{L}\right)$; and red blood cells, $0.79 \times 10^{9} / \mathrm{L}$. The glucose and LDH were normal. Ascitic fluid was negative for acid fast bacilli, for bacterial cultivated growth as well as for malignant cells. Abdominal ultrasonography: showed marked ascites and was 
otherwise unremarkable. Abdominal computed tomography (fig. 1): disclosed marked ascites and

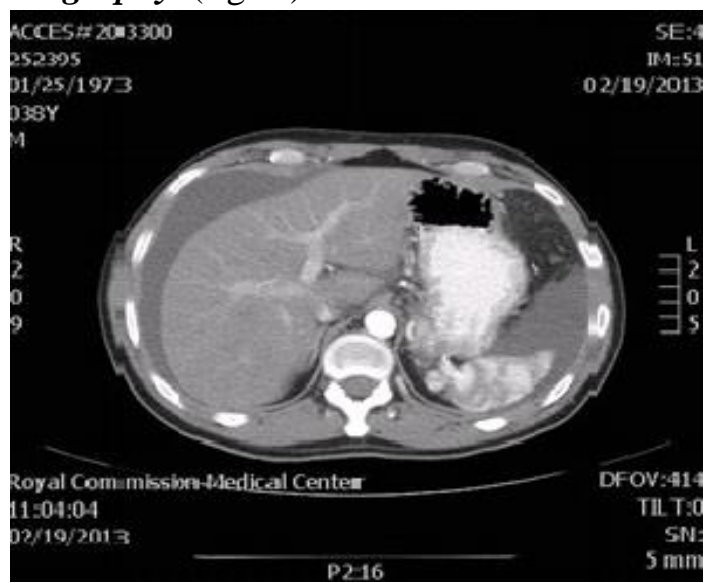

Figure 1: Ascites and gastric wall thickening in CT abdomen with eosinophils

Esophagogastroduodenoscopy: showed mild erythematous gastritis with gastric erosions, normal esophagus and normal duodenum. Multiple biopsies were taken from the esophago-gastric junction, gastric antrum, and duodenal bulb. The histopathologic examination revealed gastric mucosal infiltration with eosinophils as well as scattered plasma cells and lymphocytes (fig. 2). Helicobacter pylori organisms were not seen in the specimen. Colonoscopy: showed erythematous patches in the sigmoid colon and cecum, and multiple biopsies were taken. The histopathology reported colitis with eosinophilic infiltration of the colonic mucosa. The diagnosis of mucosal and subserosal EGE was made, depending on the above clinical, laboratory, radiologic and endoscopic findings, and histopathologic confirmation.

The patient was treated with oral prednisolone with a starting dose of $60 \mathrm{mg} /$ day, with dramatic improvement of symptoms, mainly disappearance of pain and regain of appetite, within few days. After three weeks, eosinophilic count was back to normal and there was complete resolution of ascites. Prednisolone was then tapered off gradually and completely stopped three months later. During regular follow up for the next 12 months, the patient was pain-free, with no relapse of ascites or eosinophilia.

\section{DISCUSSION AND REVIEW OF LITERATURE}

Eosinophilic gastroenteritis was first described by Kaijer in 1937, as a disease characterized by gastric antral wall thickening, and was otherwise unremarkable.

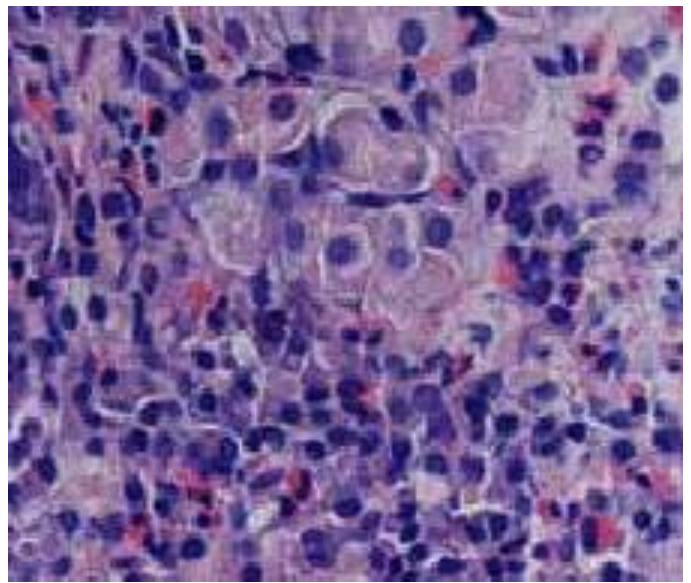

Figure 2: Infiltration of gastric mucosa

abnormal eosinophilic infiltrate in the stomach and intestine. $^{3}$ Landres in 1978 reported a case of a child with an eosinophilic infiltrate exclusively localized in esophagus. ${ }^{4}$ Since then, many reports appeared in the literature describing patients with eosinophilic infiltrates affecting different parts of the gastrointestinal tract (GIT), from the esophagus to the rectum. Rothenberg (2004) has classified these so called EGID, into EGE, eosinophilic esophagitis (EE), and eosinophilic colitis (EC), which can occur both in isolation or in associations. Each of these entities seems to have some pathological peculiarities. ${ }^{5}$ This review deals exclusively with EGE.

Etiology and pathogenesis:

The etiology of EGE is unknown, but the pathogenesis seems to be linked to an atopic predisposition, this is due to the frequent association with a personal or family history of allergies and the common finding of high levels of $\operatorname{IgE}$ and eosinophil cationic protein (ECP). The hypothesis is that eosinophils may cause damage to the gastrointestinal wall with a mechanism similar to that found in the epithelium of respiratory tract of patients with asthma. The gastrointestinal tract is the largest non-hematopoietic system where eosinophils are present in physiological conditions, residing in the lamina propria of all segments, for exception of the esophagus where they are normally absent. ${ }^{6}$

What induces tissue damage is not known, but the most accepted hypothesis is that of an external allergen. In a patient genetically prepared with 
altered intestinal permeability, an antigen reacts with surface Ig-E linked to the receptors of mast cells through the mucosa, inducing their degranulation and subsequent release of mediators such as histamine, platelet-activating factor (PAF) and leukotriene B4 (LTB4), which attract and activate the eosinophils in situ. The granules of eosinophils contain several pro-cationic proteins, such as major basic protein, the ECP, the eosinophil-derived neurotoxin and eosinophil peroxidase, which induce both a tissue damage that direct the synthesis of leukotrienes, which in turn induce the degranulation of other mast cells. ${ }^{7}$ Furthermore, eosinophils release a variety of leukotrienes and cytokines, such as interleukin-3 (IL-3), IL-5, and granulocyte macrophage - colony stimulating factor (GM-CSF), these mediators together contribute to a vicious circle with persistent infiltration of eosinophils into the gastrointestinal tract wall. The IL-5 is the most specific stimulating factor for proliferation and maturation of eosinophils in the bone marrow and inducing their release into the blood stream and attracting them into the inflammatory site. ${ }^{8}$

\section{Clinical features of EGE:}

EGE affects both children and adults, with a peak prevalence in the third and fourth decades of life. Most of the reported cases concern Caucasian populations, with a slight predominance in males. ${ }^{9}$ The clinical presentation is very heterogeneous, depending not only on the affected site (stomach and intestine with or without involvement of esophagus and colon), but also on the layer of the intestinal wall more involved. Klein in 1970 proposed the division in three forms: mucosal, muscular and subserosal. ${ }^{10}$ The mucosal form is the most common, characterized by surface infiltration, which causes "luminal" manifestations such as abdominal pain, vomiting, diarrhea, weight loss, malabsorption, iron-deficiency anemia. These patients generally have a personal or family history of allergy in about $50 \%$ of cases. In the muscular form, eosinophilic infiltration of the muscularis propria often involves the gastric antrum, and affected patients can present with pyloric or smallbowel obstruction. Less common is the subserosal form, where eosinophilic ascites develops. These patients often have an involvement of the whole wall thickness and commonly have a personal history. ${ }^{11}$ Moreover, some abnormal presentations of EGE were reported in literature, e.g. cases of acute pancreatitis, cholangitis, gastric antral ulceration and duodenal ulcers. The natural history of these diseases is in fact characterized by frequent recurrences, especially in young patients that appear to be at greater risk. ${ }^{9}$

\section{Diagnosis of EGE:}

The diagnostic criteria of EGE include demonstration of eosinophilic infiltration of the gut wall, lack of evidence of extra intestinal disease and exclusion of other causes of peripheral eosinophilia. $^{2}$ The diagnosis necessarily requires histological (biopsy or surgical specimen) or cytologic (ascitic fluid) evidence of abnormal eosinophilic infiltrate. The standardized diagnostic cut-off value, above which EGE is diagnosed, is eosinophilic count of 20/ high power field (HPF). ${ }^{5,12}$ The difficulty in the diagnosis lies not only in the fact that it could be histologically missed due to "Patchy" pathological distribution, but also in the absence of particularly suggestive endoscopic appearance. The macroscopic findings vary from a seemingly normal mucosa up to erythema, nodules and ulcerations. This is why, multiple biopsies are recommended even from apparently healthy mucosa if clinical suspicion of EGE is there. The imaging examinations often reveal nonspecific findings, the most frequent is the thickening of the gastrointestinal walls or gastric folds, more rarely, ascites can be observed. ${ }^{9,12}$ Technetium scintigraphy has also been proposed for diagnosis of eosinophilic infiltrates, but this method does not distinguish EGE from other causes of gastrointestinal inflammation. The laboratory findings are not specific. The eosinophilia in peripheral blood is absent in about $20 \%$ of the cases and the indices of inflammation are not necessarily altered. ${ }^{9,12}$ In cases of malabsorption there may be iron deficiency anemia, hypoalbuminemia and/or hypogammaglobulinemia. Allergy testing, including the skin prick test and total IgE level, might be positive. The differential diagnosis is especially with intestinal parasitic infestation or the idiopathic hypereosinophilic syndrome, i.e. the condition characterized by peripheral blood eosinophilia $>1.5 \times 10^{9} / \mathrm{L}$, for more than six consecutive months, with involvement of multiple extra-intestinal organs as skin, heart, lungs and nervous system. ${ }^{13}$ The possibility of intestinal eosinophilic infiltrate secondary to other diseases as connective tissue disorders, vasculitis, inflammatory bowel disease, lymphoma, allergies, celiac disease, 
and helicobacter pylori infection, must also be taken into account in differential diagnosis. ${ }^{5}$

\section{Treatment varieties of EGE:}

EGE is a relatively rare disease, with no available large randomized trials, and data extraction comes from case reports and small series. For these reasons, there is no unified consensus in therapy. In patients with a history of food allergy or positive SPT, as well as in those who are not steroid-responsive, an attempt of diet elimination must be considered, which however, was found only occasionally effective and burdened by frequent recurrences. ${ }^{14}$ Steroids are the drugs of choice because of the wide use and good results. ${ }^{2,9,12}$ They are typically used in regimens similar to those for chronic inflammatory diseases (i.e. $1-2 \mathrm{mg} / \mathrm{kg} /$ day for $4-8$ weeks and subsequent tapering). The effectiveness of steroids is generally rapid, but the long-term response is not guaranteed. Usually, symptoms recur after suspension or reduction of steroids, which often necessitates long-term steroid therapy or the use of immunosuppressants. ${ }^{2}$

In literature there are only few cases of effective treatment with, second-generation steroids such as budesonide and beclomethasone dipropionate. ${ }^{15}$ While many drugs have been used as steroid savers, including azathioprine, sodium cromoglycate and montelukast. ${ }^{16,17}$ Sodium cromoglycate is a stabilizer that prevents the release of toxic mediators from mast cells, and reduces the absorption of antigens from the small intestine. It has been proven effective and safe in some cases of EGE at a dose of $200 \mathrm{mg} 3-4$ times /day. ${ }^{18}$ Melamed et al. (1991) have reported a clinical-laboratory benefit in 6 patients with EGE using ketotifen, an antihistamine similar to sodium cromoglycate, at a dose of 2-4 mg/day for 1 year. ${ }^{19}$

Shirai et al. (2001) reported a successful therapeutic trial with suplatasttosilate, a new inhibitor of IL-5 and IL-4 used in asthma. ${ }^{20}$ Recently, biological therapy was attempted. The Reslizumab, a humanized anti- IL-5monoclonal antibody, has been used in four patients with EGE inducing suppression of eosinophilia and clinical symptoms. ${ }^{21}$ The Omalizumab, an anti-IgE, was used in nine patients, inducing significant decreases in peripheral eosinophilia, gastrointestinal symptoms, and a trend towards lower eosinophil number in the gastric antrum and duodenum. In contrast, there was an increase of esophageal eosinophilic infiltrate, which supports the hypothesis of a different pathophysiology between EGE and eosinophilic esophagitis. ${ }^{22}$

Surgical treatment is necessary only in cases of obstruction and/or perforation. ${ }^{2}$

\section{PROGNOSIS}

In general, the prognosis of EGE is usually good, and mortality, usually related to intestinal perforation, is very rare. ${ }^{2}$

\section{CONCLUSION}

EGE is a disease of unknown etiology increasing in clinical practice. The diagnosis is often difficult, especially in cases where the peripheral eosinophilia is absent. If the disease is suspected a broad GIT sampling is required, multiple biopsies are to be taken, even from apparently healthy mucosa. The diagnostic confirmation is in fact the histologic or cytologic evidence of inflammatory eosinophilic infiltrate in ascites fluid, after excluding all the possible causes of secondary eosinophilia. The cornerstone of therapy is steroids even if there are frequent recurrences on suspension or reduction of therapy.

\section{REFERENCES}

1. Hepburn IS, Sridhar S, Schade RR. Eosinophilic ascites, an unusual presentation of eosinophilic gastroenteritis: A case report and review. World J Gastrointest Pathophysiol 2010 Dec; 1(5):166-70. doi: 10.4291/wjgp.v1.i5.166.

2. Ingle $\mathrm{SB}$, Hinge Ingle $\mathrm{CR}$. Eosinophilic gastroenteritis: an unusual type of gastroenteritis. World J Gastroenterol 2013 Aug; 19(31):5061-6. doi: 10.3748/wjg.v19.i31.5061.

3. Kaijer R. Zur Kenntnis der allergischen Affektionen des Verdauungskanals vom Standpunkt des Chirurgen aus. Arch Klin Chir 1937; 188:36-64. Quoted from von Wattenwyl F, Zimmermann A, Netzer P. Synchronous first manifestation of an idiopathic eosinophilic gastroenteritis and bronchial asthma. Eur J Gastroenterol Hepatol 2001 Jun; 13(6):721-5.

4. Landres RT, Kuster GG, Strum WB. Eosinophilic esophagitis in a patient with vigorous achalasia. Gastroenterology 1978; 74 (6):1298-1301.

5. Rothenberg ME. Eosinophilic gastrointestinal disorders (EGID). J Allergy Clin Immunol 2004; 113:11-28.

6. Kita H. Eosinophils: multifaceted biological properties and roles in health and disease. Immunol Rev 2011; 242:161-77.

7. Masterson JC, Furuta GT, Lee JJ. Update on clinical and immunological features of eosinophilic gastrointestinal diseases. Curr Opin Gastroenterol 2011; 27:515-22.

8. Hogan SP, Rosenberg HF, Moqbel R, Phipps S, Foster PS, Lacy P, Kay AB, Rothenberg ME. 
Eosinophils: biological properties and role in health and disease. Clin Exp Allergy 2008; 38:709-50.

9. Zhang L, Duan L, Ding S, Lu J, Jin Z, Cui R, McNutt M, Wang A. Eosinophilic gastroenteritis: clinical manifestations and morphological characteristics, a retrospective study of 42 patients. Scand J Gastroenterol 2011; 46:1074-80.

10. Lucendo AJ, Arias A. Eosinophilic gastroenteritis: an update. Expert Rev Gastroenterol Hepatol 2012; 6:591-601.

11. Antonini F, Saltarelli P, Frieri G, Latella G. Eosinophilic ascites. J Gastroenterol Hepatol 2012; 27(11): 1759.

12. Talley NJ, Shorter RG, Phillips SF, Zinsmeister AR. Eosinophilic gastroenteritis: a clinico-pathological study of patients with disease of the mucosa, muscle layer, and subserosal tissues. Gut 1990; 31:54-58.

13. Cogan E, Roufosse F. Clinical management of the hypereosinophilic syndromes. Expert Rev Hematol 2012; 5:275-89.

14. Oh HE, Chetty R. Eosinophilic gastroenteritis: a review. J Gastroenterol. 2008; 43:741-50.

15. Siewert E, Lammert F, Koppitz P, Schmidt T, Matern S. Eosinophilic gastroenteritis with severe proteinlosing enteropathy: successful treatment with budesonide. Dig Liver Dis 2006; 38:55-59.

16. Pineton de Chambrun G, Gonzalez F, Canva JY, Gonzalez S, Houssin L, Desreumaux P, Cortot A, Colombel JF. Natural history of eosinophilic gastroenteritis. Clin Gastroenterol Hepatol. 2011; 9:950-956.

17. Filik L. Montelukast and maintenance of steroidinduced remission in eosinophilic esophagitis. Dig Dis Sci 2012; 57:258-9.
18. Moots RJ, Prouse P, Gumpel IM. Near fatal eosinophilic gastroenteritis responding to oral sodium cromoglycate. Gut 1995; 29:1282-1285.

19. Melamed I, Feanny SJ, Sherman PM, Roifman CM. Benefit of ketotifen in patients with eosinophilic gastroenteritis. Am J Med 1991; 90:310-314.

20. Shirai T, Hashimoto D, Suzuki K, Osawa S, Aonahata M, Chida K, Nakamura H. Successful treatment of eosinophilic gastroenteritis with suplatasttosilate. J Allergy Clin Immunol 2001; 107:924-925.

21. Wechsler ME, Fulkerson PC, Bochner BS, Gauvreau GM, Gleich GJ, Henkel T, Kolbeck R, Mathur SK, Ortega H, Patel J, Prussin C, Renzi P, Rothenberg ME, Roufosse F, Simon D, Simon HU, Wardlaw A, Weller PF, Klion AD. Novel targeted therapies for eosinophilic disorders. J Allergy Clin Immunol 2012; 130:563-71.

22. Foroughi S, Foster B, Kim N, Bernardino LB, Scott LM, Hamilton RG, Metcalfe DD, Mannon PJ, Prussin C. Anti-IgE treatment of eosinophilassociated gastrointestinal disorders. J Allergy Clin Immunol 2007; 120:594-601.

\section{ACKNOWLEDGMENT}

Great thanks to the patient who accepted to publish this case report, as well as to the histopathologist and nursing staff for their appreciated efforts and help to carry out this work. 